Article

\title{
Analysis of Existing Thermodynamic Models of the Liquid Drop Deposited on the Substrate-A Sufficient Condition of the Minimum Free Energy of the System
}

\author{
Slawomir Torbus ${ }^{1}$, Miroslaw Dolata ${ }^{2}{ }^{-}$, Slawomir Jakiela ${ }^{2}\left(\mathbb{D}\right.$ and Jacek A. Michalski ${ }^{1, *(\mathbb{C}}$ \\ 1 Faculty of Civil Engineering, Mechanics and Petrochemistry, Institute of Chemistry, Warsaw University of \\ Technology, Ignacego Lukasiewicza 17, 09-400 Plock, Poland; Slawomir.Torbus@pw.edu.pl \\ 2 Department of Physics and Biophysics, Institute of Biology, Warsaw University of Life Sciences, \\ Nowoursynowska 159, Building 34, 02-776 Warsaw, Poland; Miroslaw_Dolata@sggw.pl (M.D.); \\ Slawomir_Jakiela@sggw.pl (S.J.) \\ * Correspondence: Jacek.Michalski@pw.edu.pl; Tel.: +48-24-367-2193
}

Received: 22 October 2019; Accepted: 22 November 2019; Published: 25 November 2019

\begin{abstract}
On the basis of the principles of non-equilibrium thermodynamics, the following condition was determined: necessary and sufficient for the occurrence of a minimum free energy of a liquid droplet deposited on a solid substrate in a gaseous environment in an isothermal and isochoric system. Only for positive values of the energy of three-phase tension line (shrinking the wetting circumference) for small and large contact angles can the system not reach this minimum. Without exceeding a certain free energy limit, it is not possible for the drop to spontaneously spread over the surface. For zero and negative energy of three-phase tension line (stretching the wetting circumference), the system can always reach a minimum of free energy. The developed equations allow determining the change of free energy occurring between any two stationary states when the droplet volume and physicochemical parameters characterizing energies at the interfaces are known. For a known set of such parameters, the equations allow determining the trajectory of free energy changes in the system as a function of the contact angle from the moment the drop comes into contact with the substrate. The application of the principles of non-equilibrium thermodynamics makes it possible to treat a real system as one in which the drops do not evaporate. However, the system has to be isothermal.
\end{abstract}

Keywords: wetting angle; sessile droplet; spherical droplet; Young equation

\section{Introduction}

The phenomenon of wetting a solid substrate with liquids has been used in technical devices for centuries. Initially, the highest interest among engineers was the rheology of liquids and adhesion phenomena regarding the ability to create their uniform thin layers on solid surfaces-painting and lubrication and then spray painting and coating. At the end of the twentieth century, owing to the techniques of creating computer printouts, intensive work began on depositing on solid substrates and drops' evaporation-inkjet printers. It seems that, at the moment, special attention should be paid to the shape and stability of drops created on solid surfaces, that is, issues related to 3D printing, in a way preparing some background for the description of the behaviour of liquid drops created and flowing in microfluidic systems. It should be noted that additive manufacturing methods (3D) are increasingly applied by the industry; in turn, microfluidic devices are increasingly used in bio-, chemical, and medical analyses.

At the beginning of the 19th century, Young formulated in words the balance of forces acting at the interface of three phases, that is, a solid surface, liquid, and gas [1]. On this basis, he determined 
the contact angle as a measure of interaction between these phases. This parameter is dependent on the physical properties characterizing the interfaces between the pairs of all phases present in the system. At the same time, the concept of a potential function and fundamental differential equations describing the parameters of the phase surrounded by convex (or concave) interface are formulated [2]. It was shown that the pressure inside the convex phase should be greater than that inside the second phase surrounding it.

The Laplace equation was solved at the end of the nineteenth century, irrefutably proving the spherical shape of drops in the absence of external forces acting on them [3]. However, for drops of a given diameter, it was not yet possible to determine the pressure prevailing in the liquid phase and its value was fitted experimentally. The expression, now known as the Laplace-Young equation, appeared in literature only at the beginning of the 20th century [4]. It defines the dependence of the contact angle on the unit surface energies (equal to surface tensions), characterizing the interfaces between all pairs of phases present in the system.

On the basis of measurements of the contact angle of gas bubbles deposited on the solid substrate and surrounded by a liquid, a hypothesis assuming the presence of an additional force acting on the curved line formed by the contact of three phases—liquid, gas, and solid [5]—was introduced. The hypothesis was formulated according to Gibbs' suggestions and per analogiam to the solution of the Laplace equation, indicating the need for a pressure difference between phases separated by a curved interfacial interface. In the same year, Wenzel published a work in which he showed the effect of surface roughness on the value of the measured wetting angle [6]. He observed this phenomenon even when the liquid adhered tightly to the wetted surface. On the basis of a statistically determined parameter (roughness ratio), he empirically modified Young's equation. A slightly different approach to the description of wetting of hydrophobic and porous surfaces was applied by Cassie and Baxter [7]. They assumed that, when applying drops to the surface, the air remains trapped inside the pores. As a result, the liquid only wets the outermost surface of the solid, and stays in contact with the gas above the pores, creating a non-curved (flat) interfacial surface. According to the analysis of surface energy of the bottom part of the drops, two coefficients characterizing the geometry of the considered system were introduced into Young's equation.

The first theoretical solution [8] emerged only in 1965 and concerned the work of the formation a spherical, liquid drop surrounded by gas. The derivation for the system without the influence of external forces and without the presence of a solid phase was performed by balancing the free energy (Helmholtz) of both phases, taking into account the energy of the interface. A few years later, in the theoretical way, the influence of gravity on the shape of a deposited drop began to be analyzed [9]. However, only the energy of the liquid-gas interfacial surface and the gravitational potential energy of the drop were considered in the energy balance. Minimization of the formulated energy balance allowed to graphically determine the interdependences of geometric parameters describing the drop shape. The results of the later experiments showed an increase in the contact angle with increasing drop diameter, which is linked with exceeding of a certain size of the drops needed to stabilize the contact angle [10].

Schwartz's work was a review of the previous results of wettability investigations and pointed to the necessity of performing molecular analyses of phenomena occurring at the interfaces, in particular along the three phases contact line [11]. For this reason, its author should be treated as a precursor of research related to the molecular thermodynamics of surface phenomena associated with the wettability of solid surfaces. Besides, this work pointed to the necessity to apply thermodynamic principles to describe the behaviour of liquid drops.

On the basis of thermodynamic considerations, the equation balancing the internal energy of a system composed of the phases of gas, liquid, and solid surface was formulated [12]. The energy of particular components of the system was taken into account, that is, phases, interface surfaces, and lines of contact of three phases, and even dividing points constituting the ending of these lines. The model thus formulated would be an excellent tool for determining the internal energy of a system consisting 
of a droplet deposited on a solid substrate, both being in contact with the gas phase. Unfortunately, determining the necessary condition for thermodynamic equilibrium would require determining the exact differential of the internal energy of the system and equating it to zero. This in turn would require that the entropy values of all elements making up the system (phases, interfaces, three phase contact lines, and dividing points) be identical. This condition significantly complicates calculations. Moreover, probably starting from the assumption that the real thermodynamic system always reaches the minimum of internal energy, the equations describing its sufficient condition were neither discussed nor formulated. However, this is the only way to verify the correctness of the assumptions introduced concerning the needs of thermodynamic equilibrium achievement of the system.

The minimization of the equations of free energy (Helmholtz) balance formulated for an isothermal three-phase system is much simpler, because, by definition, there is no need to consider the entropy of the system components $[13,14]$. In the obtained equation of the free energy balance of the spherical drop, according to the equation developed in Reiss's monograph [8], terms describing the free energy of both fluid phases were equal to zero. The final result was Young's equation modified by the term corresponding to the interaction of the three-phase contact line, in which the characteristic dimension was the radius of the circle constituting the liquid-solid interface. The solution of the model equations [13] was performed using the principles of classical thermodynamics, assuming the thermodynamic equilibrium of the system, that is, without the need to discuss the effect of drop evaporation (Kelvin effect) on its volume. An identical result, based on the same assumptions, was presented in an article almost simultaneously published by Widom [15]. As a result of the adoption of the assumption of thermodynamic equilibrium, it was impossible to perform a full discussion of solutions indicating the possibility of non-equilibrium behaviour of the considered system. Therefore, in the work of [15], only information in words was given, such that non-equilibrium conditions may occur. In none of the presented papers [13-15] was the sufficient condition of the minimum free energy of the system formulated. The range of variation of physicochemical parameters was also not provided, which would promote the transition from the equilibrium state to the thermodynamic non-equilibrium state.

It should be stressed that the equation obtained in this way is the fundamental relationship applied in many works [16-20], with the dummy assumption that free energy can reach a minimum in every system considered; that is, its second derivative should be positive. It should be noted that the correctness of determining the contact angle under thermodynamic equilibrium conditions (minimum free energy of the system) also affects the description of phenomena related to mass and heat exchange during drops' evaporation [21,22].

In all cited papers, the drops were assumed to be large enough to be considered as a continuous phase (continuum). On the experimental basis [13,14], it is known that this approach is appropriate for drops with a volume greater than about $0.01 \mu \mathrm{L}$. The applied equations (Young's and Laplace-Young) are also met in these conditions, and even for much larger drops when they begin to deform as a result of gravitational force [23].

Schwartz's work [11] was most likely an inspiration to start the simulation of the wetting phenomenon based on the interactions of the molecules in the system. The function of Lennard-Jones potential [24] was used for describing the interactions between liquid molecules, as well as liquid molecules with solid phase molecules. The result of the simulation being the angle between the tangent to the boundary of the liquid-vapour interface (at the point of contact of the three phases) to the solid surface was compared with the wetting angle determined for the macroscopic system from Young's equation. Owing to numerical limitations, the first works addressed the issue of meniscus as a two-dimensional system $[25,26]$. Unfortunately, the results of these works were not satisfactory and showed a large discrepancy between the macroscopic and molecular systems.

After introducing the dependence of tension in the three-phase tension line on the curvature of the wetted surface (Tolman equation) [27,28], the compatibility of the results of molecular computer simulations with Young's equation was significantly improved. However, the above relationship 
becomes negligible for deposited droplets as soon as their wetting radius exceeds a few nanometers [28]. Also, on the basis of the results presented in another paper [28], it can be estimated that the influence of the curvature of the liquid-vapour interface on the surface tension becomes negligible for its sum of major curvatures greater than $2.5 \times 10^{6} \mathrm{~m}^{-1}$, which corresponds to the radius of spherical droplet of 80 $\mathrm{nm}\left(2.14 \times 10^{-9} \mu \mathrm{L}\right)$.

It seems that the main barrier to performing molecular simulations is still the low computing power of the computers applied. In several decades, the number of liquid molecules and molecules constituting the surface of a solid substrate participating in the simulation increased from a few hundred to about $2 \times 10^{4}$. This corresponds to the volume of liquid (water) as little as $6 \times 10^{-10} \mu \mathrm{L}$, which shows that there is still a very large gap between the two methods of description of the system under consideration.

The aim of the work was to theoretically determine the trajectory of changes in the free energy of the isochoric and isothermal system during the deposition of a spherical drop of liquid on the ideal, isotropic surface of the solid substrate, and determination of the necessary and sufficient conditions for the achievement of the minimum of this energy. A continuum system not influenced by external forces was considered.

The text of the entire work attempted to apply, as far as possible, the terminology proposed in one of the last publications [29].

\section{Model Formulation}

In the general case, according to the properties of the state function, the change of the free energy (Helmholtz) of the system during the thermodynamic process from the initial state (index I) to the final state (index F) is described as follows:

$$
F=F_{F}-F_{I}
$$

This basic equation is given because, in many thermodynamic models, the initial state of the system is skipped. In theoretical papers [12], the lack of this information is often obvious; however, in both theoretical and experimental papers [13-16], this state may determine one of the limits that constitute the range of applicability of the model.

Let us perform the deposition and spreading of liquid droplet on the surface of a solid substrate surrounded by a gas (vapour) as an isothermal process $(T=$ const). This means that, in each of these two states (initial and final), it is possible to describe the free energy of the system with the following equation:

$$
\begin{aligned}
F_{i}=-p_{L} V_{L}-p_{G} V_{G}+\sigma_{S L} a_{S L} & +\sigma_{S G} a_{S G}+\sigma_{L G} a_{L G}+\gamma_{S L G} \Lambda_{S L G}+\mu_{L} n_{L}+\mu_{G} n_{G}+\mu_{S L} n_{S L} \\
& +\mu_{S G} n_{S G}+\mu_{L G} n_{L G}+\prod
\end{aligned}
$$

Let us discuss the components of $F_{i}$ in Equation (2):

(a) The first component denotes energy accumulated in the volume of liquid $V_{L}$ under pressure $p_{L}$ (from the definition of free energy, it must have a negative value).

(b) The second component denotes the energy accumulated in the gas volume $V_{G}$ under pressure $p_{G}$ (from the definition of free energy, it must have a negative value).

(c) The third, fourth, and fifth components denote surface energy (on the phase boundaries); that is, on the contact surface of solid and liquid (index $S L$ ), on the contact surface of solid and gas (index $S G$ ), and on the surface of liquid and gas contact (index $L G$ ), respectively. $\sigma$ denotes the unit energy (surface tension) of indexed interfacial surface, and $a$ is its surface area.

(d) The sixth component describes the energy sourcing from the three-phase contact line, and it is the product of the unit energy of this line $\left(\gamma_{S L G}\right)$ (energy per length unit) and its length $\left(\Lambda_{S L G}\right)$.

(e) The seventh and eighth components describe the chemical potentials "accumulated" in the liquid and gas phases, respectively. However, using the zeroth law of thermodynamics (the condition of 
the system equilibrium), one can assume the infertility of both phases, and these terms can be assumed to be equal to zero.

(f) The ninth to eleventh components describe the chemical potentials occurring at the phase boundaries. Here too, using the zeroth law of thermodynamics (system equilibrium condition), one can assume the lack of mutual reactivity of solid, liquid, and gas components, and consequently assume the zero value of these terms.

(g) The twelfth component refers to "other" energy accumulated in the system or at the interfaces. This other energy will appear in the case of acting on the system of forces of external fields, for example, gravitational, electrostatic, and magnetic. Then, in place of parameter $\prod$ one should write the appropriate mathematical expression describing a given type of interactions. However, there are no external force fields in the system under consideration; therefore, the term is equal to zero.

Taking into account the results of the above discussion, Equation (2) describing the free energy of a system consisting of a drop of liquid, solid surface, and gas phase will be simplified to the following form:

$$
F_{i}=-p_{L} V_{L}-p_{G} V_{G}+\sigma_{S L} a_{S L}+\sigma_{S G} a_{S G}+\sigma_{L G} a_{L G}+\gamma_{S L G} \Lambda_{S L G}
$$

In the papers cited in the introduction describing thermodynamic models of settlement and spreading of liquid drops on the surface of a solid substrate $[12-16,18,19]$, the volume of the liquid phase during the thermodynamic process is unchanged. It is tantamount to a silent assumption of incompressibility and non-evaporation of the liquid. This means that, at a constant gas pressure, the three-phase thermodynamic system is considered as isochoric, and hence its volume $\left(V_{T}\right)$ is equal to the following:

$$
V_{T}=V_{L}+V_{G}=\text { cnnst }
$$

It should be noted that the information presented in the experimental papers, showing that, in the experiments carried out in the open system, the drop did not change its volume, does not determine either the thermodynamic equilibrium or the isothermal nature of such a system. This is evidenced by the results of the experiments presented in the work of [21]. They show that, in the case of macroscopic drops (50 $\mu \mathrm{L}$-water), the temperature difference between the drop base and the liquid-gas surface can reach as much as $10 \mathrm{~K}$. This is because of the outflow of heat (evaporation) along with the vapour stream. Such a large difference in temperature has a noticeable effect on surface tensions at the liquid-vapour and liquid-solid interfaces.

The surface of the solid substrate present in the considered system has its finite value $\left(a_{T}\right)$, although it is sufficiently large that the liquid can wet it without mechanical obstacles caused by, for example, the occurrence of edges. Thus, in the general case of an ideal and isotropic surface, the following relationship will be fulfilled:

$$
a_{S L}+a_{S G}=a_{T}=\text { cnnst. }
$$

After substituting Equations (4) and (5) into Equation (3), we get the following:

$$
F_{i}=-\left(p_{L}-p_{G}\right) V_{L}+\left(\sigma_{S L}-\sigma_{S G}\right) a_{S L}+\sigma_{L G} a_{L G}+\gamma_{S L G} \Lambda_{S L G}-p_{G} V_{T}+\sigma_{S G} a_{T} .
$$

In all previously published thermodynamic models of drops deposited on solid substrate $[13,15$, 30-33], the first term, in Equation (6), is equated to zero, which, in the case of a levitating drop in gas, is consistent with the theoretical solution [8]. This approach is also supported by the need to maintain zero work value resulting from the change in phase volumes in the process performed in the isochoric system (1). However, it should be noted that we do not discuss here the validity of this assumption, but only introduce it because of its wide application, such as in the work of [31].

The last two components, in Equation (6), assume constant and the same values in the initial and final states of the considered thermodynamic process. 
Considering the above discussion and Equation (1), the dependence (6) can be simplified to the following form:

$$
F_{i}=\left(\sigma_{S L}-\sigma_{S G}\right) a_{S L}+\sigma_{L G} a_{L G}+\gamma_{S L G} \Lambda_{S L G}
$$

The second assumption concerns the geometry of the system, and more precisely, the shape of the liquid-gas interface. In each case analyzed in the literature, the axial symmetry of the drop is assumed [12-16,18,19,30-33]. In the absence of external forces acting on the system and for the isotropic surface of the substrate, a spherical drop shape is assumed. Figure 1 gives the designation of dimensions used in several of the following equations:

(a) radius of drop base:

$$
\alpha=R \sin \varphi
$$

(b) drop height:

$$
z=R(1-\cos \varphi)
$$

where $R$ denotes radius of spherical drop cup and $\varphi$ is contact angle.

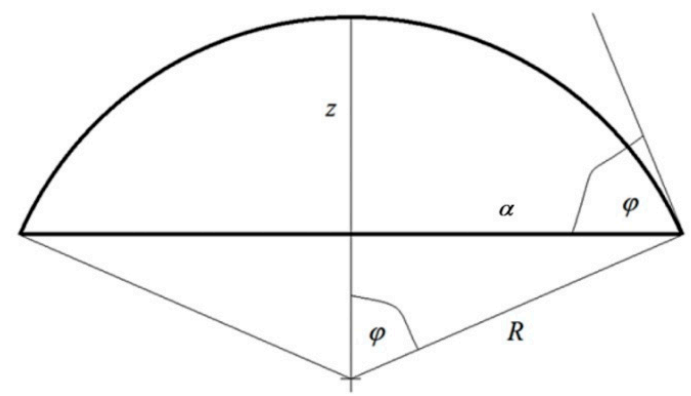

Figure 1. The assumed notation of geometric drop dimensions.

Consequently, the expressions in Equation (7) are defined by the following relations:

(a) interfacial surface area of solid substrate-liquid:

$$
a_{S L}=\pi R^{2} \sin ^{2} \varphi
$$

(b) area of liquid-gas interface:

$$
a_{L G}=2 \pi R^{2}(1-\cos \varphi)
$$

(c) the length of the three phase tension line between solid-liquid-gas:

$$
\Lambda_{S L G}=2 \pi R \sin \varphi
$$

To further simplify the transformations, let us express the volume of liquid in the system as the volume of a sphere with a radius $R_{\pi}$. From the fact that this volume must be equal to the volume of the section of liquid sphere deposited on a solid substrate, we obtain a relationship between the radius of this sphere section and the contact angle $\varphi$ :

$$
R=R_{\pi}\left[\frac{4}{(1-\cos \varphi)^{2}(2+\cos \varphi)}\right]^{\frac{1}{3}}
$$

This equation results from the balance of the volume (mass) of the drop spreading on the surface of the solid substrate in an isothermal system. If, according to the principles of classical thermodynamics, we assume that the drop must always be in thermodynamic equilibrium (liquid/vapour) with the 
surrounding gas, then we can get at most one state in which this equilibrium would be achieved. This will also happen when the vapour pressure of the liquid in the gas is equal to the vapour pressure on the surface of the droplet. As the drop settles on the solid surface, the curvature of the liquid-gas surface changes. Therefore, according to the condition of maintaining thermodynamic equilibrium and the Kelvin equation, the content of liquid vapours in the gas phase will change. In a closed system, as a result of condensation or evaporation of a liquid, its volume will change. By applying the principles of non-equilibrium thermodynamics, the need to consider the evaporation/condensation process can be avoided by treating drop deposition as a system transition through a series of consecutive steady states in which the volume of the liquid phase remains constant. In this case, the search for the free energy extremum of the system is changed into the search for extremum of the sum of surface energies and tension line occurring in the system. However, such a system has to be isothermal. In addition, the equations describing equilibrium and stationary systems remain unchanged. A detailed description can be found in the Appendix A.

After substituting Equation (13) into Equations (10)-(12) and entering the obtained dependencies into Equation (7), and then into Equation (1), which takes into account the simplifications used to derive Equation (7), we get the following:

$$
\begin{gathered}
F(\varphi)=\pi R_{\pi}^{2}\left[\frac{4}{(1-\cos \varphi)^{2}(2+\cos \varphi)}\right]^{\frac{2}{3}}\left(1-\cos ^{2} \varphi\right)\left\{\sigma_{S L}-\sigma_{S G}+\sigma_{L G} \frac{2}{1+\cos \varphi}\right. \\
\left.+\frac{2 \gamma_{S L G}}{R_{\pi}}\left[\frac{1}{4} \frac{(2+\cos \varphi)}{(1-\cos \varphi)}\right]^{\frac{1}{3}} \frac{\sin \varphi}{(1+\cos \varphi)}\right\}-F_{I}
\end{gathered}
$$

The above equation is the dependence of the free energy of the isothermal system on the contact angle and liquid volume in a drop $\left(R_{\pi}\right)$.

Let us consider its usefulness from the classical thermodynamic point of view. For this purpose, we will establish a hypothetical system consisting of a solid surface $a_{T}$ with a total volume of $V_{T}$ and a certain initial volume of liquid. When the thermodynamic equilibrium is reached, the gas will be saturated with liquid vapour and a drop (with a volume $V_{L}$ smaller than the initial one) on the solid substrate will be formed, characterized by a specific contact angle. Further equilibrium processes cannot be carried out, which means that the initial state of the system is also the final state. One can only minimize Equation (14) by looking for its minimum, but this problem was sketchy solved years ago $[13,15]$. Even if the second derivative of Equation (14) was determined, it would not be possible to discuss free energy variation in areas where it does not reach the minimum. It would be a contradiction because Equation (14) was derived assuming thermodynamic equilibrium. The above discussion shows that the analysis of the behaviour of deposited droplets that do not meet the conditions of thermodynamic equilibrium is not possible based on the principles of classical thermodynamics.

The application of principles of non-equilibrium thermodynamic allows one to separate the problem of the liquid-vapour equilibrium from the problem of the mechanical energy of a drop spreading on the surface of a solid substrate. The assumed, additional thermodynamic cycle (Appendix A) ensures that the droplet volume remains constant (its mass), regardless of whether the drop has reached thermodynamic equilibrium (including balance of forces acting on it). The only restriction for using this method is to preserve the isothermal state of the system. It is worth noting that this behaviour of the considered system is equivalent to the isothermal spread of a non-evaporating liquid drop. Therefore, the initial free energy of the drop $F_{I}$ may correspond to any state between the total spread of the droplet over the entire solid surface and the state where the drop contacts it only at one (mathematical) point, that is, when it begins to spread.

\section{Model Solution}

As stated in the aim of the paper, we consider all phases (liquid, gas, and solid surface) as ideal, isotropic, and continuous-continuum. Therefore, the physicochemical parameters defining their unit 
surface energies, including the unit energy of a three-phase contact line, are taken as constant values. It follows that the application of the model is limited to drops large enough to omit the influence of drop curvatures on the surface tension at the liquid-gas interface and the stress occurring along the three-phase contact line [28]. In addition, we assume that all further analyzed processes are performed for a constant (but any value) droplet volume, $R_{\pi}=$ const, and a spherical shape of its cup.

The necessary condition for the occurrence of the minimum free energy (in the view of classical thermodynamics and non-equilibrium thermodynamics) of the considered system is the zeroing of the first derivative of the Equation (14) with respect to the contact angle. The obtained equation has the following form:

$$
\begin{gathered}
F^{\prime}(\varphi)=-2 \pi R_{\pi}^{2}\left[\frac{4}{(1-\cos \varphi)^{2}(2+\cos \varphi)}\right]^{\frac{2}{3}} \frac{\sin \varphi}{2+\cos \varphi}\left\{-\left(\sigma_{S G}-\sigma_{S L}\right)+\sigma_{L G} \cos \varphi\right. \\
\left.+\frac{\gamma_{S L G}}{R_{\pi}}\left[\frac{1}{4} \frac{2+\cos \varphi}{1-\cos \varphi}\right]^{\frac{1}{3}} \frac{\sin \varphi}{1+\cos \varphi}\right\}
\end{gathered}
$$

The factor standing in front of the curly brackets is zeroing for the contact angle $\varphi=180^{\circ}$ (when the drop contacts the surface of the substrate only at one point). Meanwhile, the expression in curly brackets is equal to zero when the following equation is fulfilled:

$$
\frac{\sigma_{S G}-\sigma_{S L}}{\sigma_{L G}}=\cos \varphi+\frac{\gamma_{S L G}}{\sigma_{L G} R_{\pi}}\left[\frac{1}{4} \frac{2+\cos \varphi}{1-\cos \varphi}\right]^{\frac{1}{3}} \frac{\sin \varphi}{1+\cos \varphi}
$$

To check the correctness of the derivation, we use Equations (8) and (13), and then the above dependence will take the following form:

$$
\frac{\sigma_{S G}-\sigma_{S L}}{\sigma_{L G}}=\cos \varphi+\frac{\gamma_{S L G}}{\sigma_{L G} \alpha}
$$

Fully in agreement with Young's equation with the influence of three-phase contact line presented in the cited papers included $[13,15]$. It should be noted that, in some papers, the sign in front of the component of the energy of the three-phase contact line is negative [16-20]. This is the result of either the introduction of such an initial assumption or the effect of a simple transformation of Equation (16), caused by the introduction of a term concerning "pure" Young's equation.

It is worth noting that Widom [15] predicted the occurrence of such solutions of Equations (16) and (17), for which the sufficient condition for the minimum of Equation (14) is not fulfilled. He pointed out in words that such solution areas will be located near the contact angles $\varphi=0$ and $\varphi=180^{\circ}$ and for the positive values of parameter $\gamma_{S L G}>0$. However, by conducting an analysis based on the principles of classical thermodynamics and lack of experimental evidence showing such thermodynamically unstable systems, he could not prove it. As a result, he could not formulate equations defining the boundaries of these areas.

The sufficient condition of the minimum free energy (in view non-equilibrium thermodynamics) of the analyzed system takes place when the second derivative (with respect to $\varphi$ ) of Equation (14), that is,

$$
\begin{gathered}
F^{\prime \prime}(\varphi)=2 \pi R_{\pi}^{2}\left[\frac{4}{(1-\cos \varphi)^{2}(2+\cos \varphi)}\right]^{\frac{2}{3}} \frac{1}{(2+\cos \varphi)^{2}}\left\{-\left(\sigma_{S G}-\sigma_{S L}\right)\left(1+2 \cos \varphi+2 \cos ^{2} \varphi\right)\right. \\
+\sigma_{L G} \cos \varphi\left(1+2 \cos \varphi+2 \cos ^{2} \varphi\right)+\frac{\gamma_{S L G}}{R_{\pi}}\left[\frac{1}{4} \frac{2+\cos \varphi}{1-\cos \varphi}\right]^{\frac{1}{3}} \frac{\sin \varphi\left(1+2 \cos \varphi+2 \cos ^{2} \varphi\right)}{1+\cos \varphi} \\
\left.+\sigma_{L G}\left(1-\cos ^{2} \varphi\right)(2+\cos \varphi)-\frac{\gamma_{S L G}}{R_{\pi}}\left[\frac{1}{4} \frac{2+\cos \varphi}{1-\cos \varphi}\right]^{\frac{1}{3}} \frac{\sin \varphi}{1+\cos \varphi}\right\}
\end{gathered}
$$


takes positive values at the points where the first derivative is equal to zero:

$$
\begin{gathered}
F^{\prime \prime}(\varphi)=2 \pi R_{\pi}^{2}\left[\frac{4}{(1-\cos \varphi)^{2}(2+\cos \varphi)}\right]^{\frac{2}{3}} \frac{1}{(2+\cos \varphi)^{2}}\left\{\sigma_{L G}\left(1-\cos ^{2} \varphi\right)(2+\cos \varphi)\right. \\
\left.-\frac{\gamma_{S L G}}{R_{\pi}}\left[\frac{1}{4} \frac{2+\cos \varphi}{1-\cos \varphi}\right]^{\frac{1}{3}} \frac{\sin \varphi}{1+\cos \varphi}\right\}
\end{gathered}
$$

The last equation was obtained by substituting Equation (16) to (18). The factor standing in front of the curly brackets is positive for all values of the contact angle $\varphi$. This means that the expression included in brackets determines the sign of the second derivative. The minimum of (14) occurs when this expression is positive. The equation that limits the area in which the free energy of the system reaches the minimum from the rest can be obtained by solving the system of Equation (16) and the following:

$$
0=\left(1-\cos ^{2} \varphi\right)(2+\cos \varphi)-\frac{\gamma_{S L G}}{\sigma_{L G} R_{\pi}}\left[\frac{1}{4} \frac{2+\cos \varphi}{1-\cos \varphi}\right]^{\frac{1}{3}} \frac{\sin \varphi}{1+\cos \varphi}
$$

The obtained equation fulfils the necessary condition of the appearance of the minimum free energy of the considered system $\left(F^{\prime}(\varphi)=0\right)$ and the condition of zeroing the second derivative of the equation describing its free energy $\left(F^{\prime \prime}(\varphi)=0\right)$ :

$$
\frac{\sigma_{S G}-\sigma_{S L}}{\sigma_{L G}}=2(1+\cos \varphi)-\cos ^{2} \varphi(2+\cos \varphi)
$$

Its analysis shows that the minimum free energy (thermodynamic equilibrium) system will reach the area below the line determined by Equation (21) on the graph $\frac{\varphi_{S G}-\varphi_{S L}}{\sigma_{L G}}=f(\varphi)$

To complete the solution, a representative initial state of the considered thermodynamic process should be selected (1). It seems that the most appropriate will be one in which a spherical drop touches the solid substrate in one "mathematical point". Then, its free energy $F_{I}$ (vide (14)) will be described by the following equation:

$$
F_{I}=4 \pi R_{\pi}^{2} \sigma_{L G}
$$

After the introduction of the above expression into the dependence (14), we get an equation determining the dimensionless free energy as a function of the contact angle:

$$
\begin{aligned}
\Delta f=\frac{F(\varphi)}{\pi R_{\pi}^{2} \sigma_{L G}}= & {\left[\frac{4}{(1-\cos \varphi)^{2}(2+\cos \varphi)}\right]^{\frac{2}{3}}\left(1-\cos ^{2} \varphi\right)\left\{-\frac{\sigma_{S G}-\sigma_{S L}}{\sigma_{L G}}+\frac{2}{1+\cos \varphi}\right.} \\
& \left.+\frac{2 \gamma_{S L G}}{\sigma_{L G} R_{\pi}}\left[\frac{1}{4} \frac{(2+\cos \varphi)}{(1-\cos \varphi)}\right]^{\frac{1}{3}} \frac{\sin \varphi}{(1+\cos \varphi)}\right\}-4
\end{aligned}
$$

Then, after entering the expression (16), we get an equation to determine the dimensionless free energy of the drop at all points where its first derivative is equal to zero:

$$
\begin{gathered}
\Delta f=\frac{F(\varphi)}{\pi R_{\pi}^{2} \sigma_{L G}}=\left[\frac{4}{(1-\cos \varphi)^{2}(2+\cos \varphi)}\right]^{\frac{2}{3}}(1-\cos \varphi)\{(1-\cos \varphi)(2+\cos \varphi) \\
\left.+\frac{\gamma_{S L G}}{\sigma_{L G} R_{\pi}}\left[\frac{1}{4}(2+\cos \varphi)(1+\cos \varphi) \sin \varphi\right]^{\frac{1}{3}}\right\}-4 .
\end{gathered}
$$

\section{Results and Discussion}

Although Equation (17) has been known and used for many years to approximate experimental data, it was impossible to perform a reliable analysis of the physicochemical parameters (unit energy interfaces and unit energy of a three-phase tension line) and droplet volume on the behaviour of the considered three-phase system owing to the necessity compliance with the principles of classical thermodynamics. Only the application of the principles of non-equilibrium thermodynamics and the 
separation of the liquid-vapour equilibrium problem from the mechanical interactions of drops with the solid substrate justify such a discussion.

In the literature [34], there is a very large discrepancy between the values of the three-phase tension line calculated theoretically based on intermolecular interactions and those measured in experiments. However, the widespread use of Equation (17) to approximate the results of experiments prompts the use of experimental rather than theoretical values. In our considerations, we take into account the droplet volume greater than $0.01 \mu \mathrm{L}$ and the tension values at the three-phase tension line given in the papers, for example, in the works of $[13,14]$, and then the $G=\frac{\gamma_{S L G}}{\sigma_{L G} R_{\pi}}$ factor can reach values up to approximately \pm 5 .

Figure 2 shows the dependences of the ratio of the unit energies of interfaces $\frac{\sigma_{S G}-\sigma_{S L}}{\sigma_{L G}}$ as a function of the contact angle $\varphi$ for several unit energy values of the three-phase tension line expressed by parameter $G=\frac{\gamma_{S L G}}{\sigma_{L G} R_{\pi}}$. The graph was created on the basis of Equation (16) and a line delimiting the solution area was marked on it for which free energy of the system has a minimum-Equation (21). It is noteworthy that every point of the chart below this line meets the necessary and sufficient conditions for the minimum free system energy (thermodynamic equilibrium) - gray area.

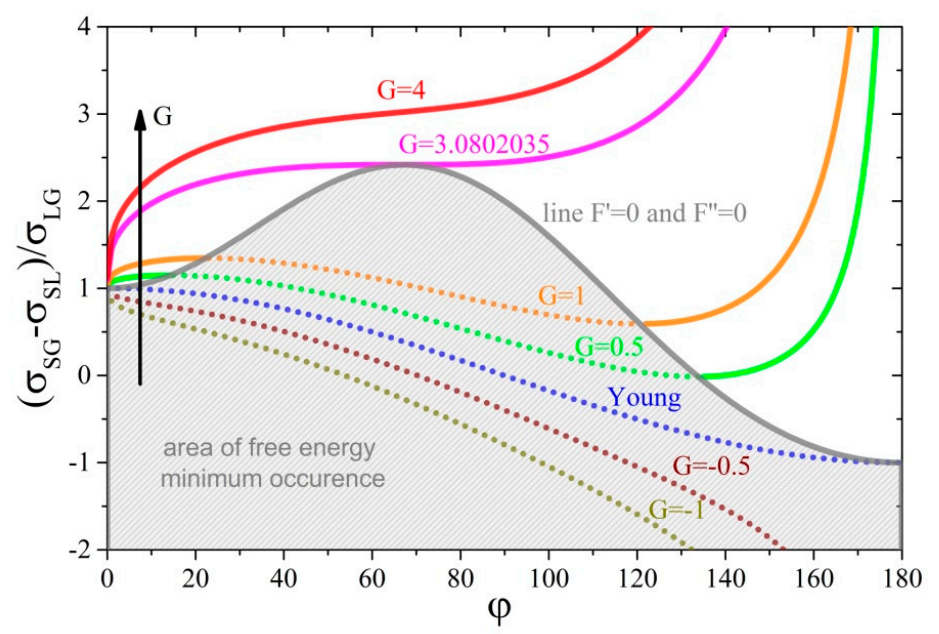

Figure 2. The dependence of the ratio of unit energies of interfaces $\frac{\sigma_{S G}-\sigma_{S L}}{\sigma_{L G}}$ versus contact angle for several values of parameter $G=\frac{\gamma_{S L G}}{\sigma_{L G} R_{\pi}}$. The area below line $F^{\prime}=0$ and $F^{\prime \prime}=0$ concerns the three-phase system reaching the minimum of its free energy (partially in view of equilibrium (gray area) and fully from a non-equilibrium thermodynamics point of view).

From the chart in Figure 2, it follows that, for the zero value of the parameter $G$, the course of the function given by Equation (16) closely corresponds to the solutions of Young's equation, and in addition, both conditions of the minimum free energy are met in the whole range of contact angle variability. This means that each of the solutions of this equation meets the conditions of thermodynamic equilibrium. As the value of this parameter decreases (for $G<0$ ), that is, when the tension in the three-phase tension line is stretching the circumference of the wetted area, the contact angle decreases. The strength of the effect of changes in the $G$ parameter on changes in the contact angle is easiest to analyze using the assumption of $\frac{\sigma_{S G}-\sigma_{S L}}{\sigma_{L G}}=$ const. While in the range of positive values of the parameter $G(G>0)$, that is, when the tension in the three phase tension line shrinks circumference of the wetted area, the contact angle increases with the increase in $G$.

For zero and negative values of parameter $G$ (drops stretched by the action of the three-phase tension line), the three-phase system under consideration is always able to reach the minimum free energy (in view of classical and non-equilibrium thermodynamics)—both conditions are always satisfied. Moreover, the graph shows that even a very high value of repelling energy of liquid from solid substrate $\frac{\sigma_{S G}-\sigma_{S L}}{\sigma_{L G}}<-1$ does not affect them. On the other hand, for positive values of parameter $G$ (drops shrank by the action of three-phase tension line), only in the middle range of variation of 
the contact angle, the considered system can achieve the minimum free energy. For small as well as large values of the contact angles, the second derivative of the free energy function (19) assumes negative values. It should be noted that reducing the volume of drops while maintaining the other physiochemical parameters will cause the transition to the area where the second derivative will be less than zero, that is, it will not satisfy the minimum free energy requirements. From experimentally measured unit energy values of three-phase tension lines [14], it follows that, in some systems, such a transition may occur even for relatively large drops with a radius of the wetted surface of even $1 \mathrm{~mm}$. Moreover, one can see from the graph in Figure 2 that, for $G>3.08$, the system is not able to reach the minimum in the free energy for any set of physicochemical parameters characterizing surface tensions at the interface surfaces or drop volume.

From the graph in Figure 2, there is one more important conclusion regarding the ranges of both small and large contact angles. Namely, for positive values of parameter $G<3.08$ and the same parameters describing interfacial surfaces and volume of $\operatorname{drop}\left(\frac{\sigma_{S G}-\sigma_{S L}}{\sigma_{L G}}=\right.$ const and $G=$ const $)$, there are at most three points (contact angles) for which the first derivative of free energy is equal to zero (necessary condition). However, only at one point does the second derivative of it take a positive value (sufficient condition), indicating the minimum of this function.

Parameter $G$ is the ratio of the force acting along the wetted circumference, being the three-phase boundary to the surface tension force acting on the surface of the spherical drop just before its deposition. Figure 2 shows that it is a measure of the deviation of the contact angle from its value determined from the "pure" Young's equation. In addition, the positive value of this coefficient shows that in, the three-phase system under consideration, there will be ranges of contact angles at which the deposited drop will not be able to reach the thermodynamic equilibrium, but will only be in the state of equilibrium of forces acting on the liquid.

It is worth investigating the dependence of the change of dimensionless free energy that occur from the point when the drop contacts the surface of the substrate until the first derivative of the free energy acquires a zero value as a function of the contact angle. The sign of such a relationship shows whether the achievement of the final state requires delivery of work to the system (positive sign) or spreading drop is a source of work (negative sign). Additionally, one can find out the amount of added or released work during such a process. This dependence was determined based on Equation (24), and its course for several values of parameter $G$ is shown in Figure 3.

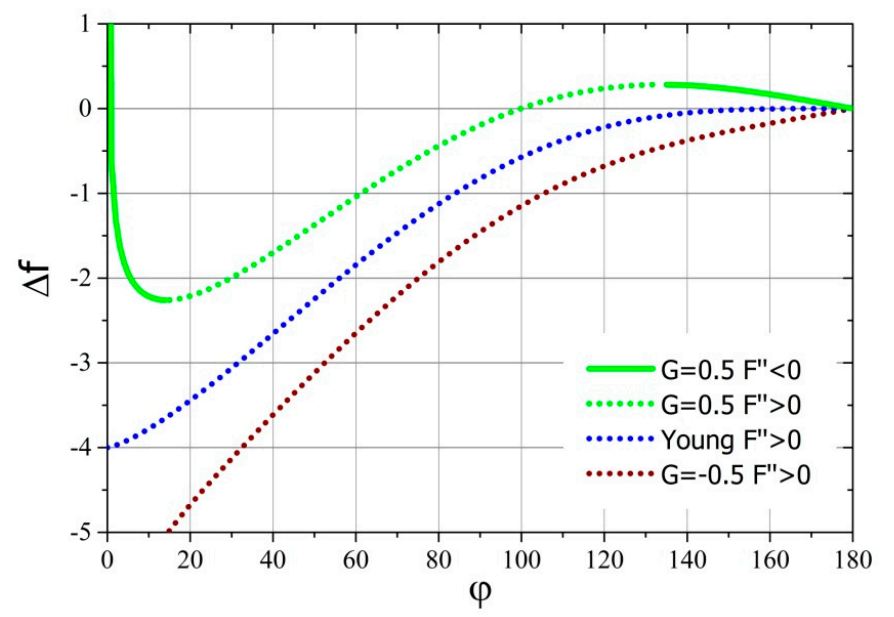

Figure 3. Dimensionless free energy of droplet deposition on the contact angle for several values of the parameter $G=\frac{\gamma_{S L G}}{\sigma_{L G} R_{\pi}}$.

From the graph in Figure 3, it follows that the dimensionless free energy of the droplet deposition monotonically decreases with the decrease of the equilibrium contact angle (see Figure 2) of the drop deposited on a solid substrate for negative and zero values of the parameter $G=\frac{\gamma_{S L G}}{\sigma_{L G} R_{\pi}}$. However, 
for positive values of the parameter $G$, the free energy of drop deposition will initially increase with the decrease of final contact angle. This increase will continue until reaching the local maximum free energy; however, up to this point, the drop will not reach thermodynamic equilibrium. After exceeding this point, the free energy of the system will decrease to the local minimum, and then it will grow to achieve asymptotic - the infinite value for the contact angle equal to zero. It should be noted that only between this maximum and minimum is the drop able to achieve thermodynamic equilibrium.

In the discussed case, the equilibrium system (classic thermodynamics) was replaced by a stationary system (non-equilibrium thermodynamics); however, the equations defining free energy in both systems did not change in their integral (6) and (7) or differential (A25) forms. Therefore, the properties regarding the changes of the analyzed free energy in processes will be the same in both systems. This has already been used "silently" above to discuss the work of drop deposition. Thus, it is easy to see that, for the zero and negative values of parameter $G$, the process of spreading the drop from the moment of contact with the substrate is always spontaneous $(\Delta f<0)$. On the other hand, in the case of positive values of the parameter $G$, a certain amount of free energy has to be initially provided in order to deposit the drop on the surface $(\Delta f>0)-a$ forced thermodynamic process. Only after exceeding the local maximum of free energy, the spread of the drop on the solid substrate will occur spontaneously, until the local minimum is reached. After its passage, the drop will spread on the surface of the substrate only when free energy (work, for example, kinetic or gravitational potential energy of the droplet) is supplied to it.

Owing to the above observations, it is worth discussing the case of the drop deposition in the system for which we choose a set of physicochemical parameters arbitrarily, for example, $\frac{\sigma_{S G}-\sigma_{S L}}{\sigma_{L G}}=0.5$ and $G=\frac{\gamma_{S L G}}{\sigma_{L G} R_{\pi}}=0.5$. This kind of analysis will be performed on the basis of the plot of Equation (23), which is shown in Figure 4. It is noteworthy that the graph created will constitute the trajectory of the free energy changes of a precisely defined system during the gradual and stationary (quasi-equilibrium) spreading of the drop over the substrate.

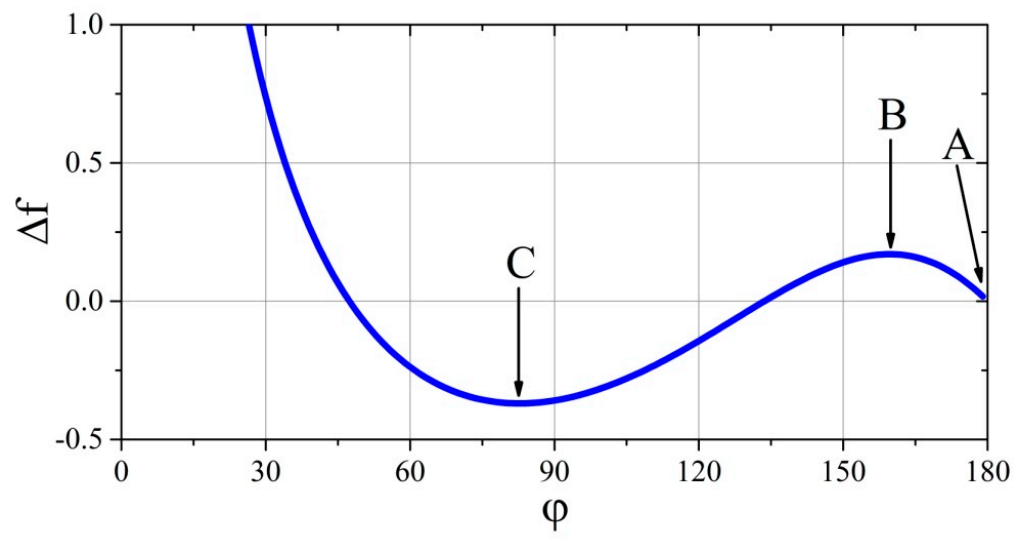

Figure 4. Trajectory of changes of dimensionless free energy of a system characterized by the following physicochemical parameters: $\frac{\sigma_{S G}-\sigma_{S L}}{\sigma_{L G}}=0.5$ and $G=\frac{\gamma_{S L G}}{\sigma_{L G} R_{\pi}}=0.5$.

Let consider the case when the drop initially touches the substrate with the contact angle $\varphi=180^{\circ}$-point $\mathrm{A}$ in Figure 4. In this case, the positive values of parameter $G$ and no influence of external force fields prevent the spread (reduce $\varphi$ ), because of the lack of free energy needed to exceed the free energy of point B. Free energy at this point is a some type of energy barrier, beyond which the spherical drop on the substrate could spread spontaneously, and the system could reach a minimum of free energy (point $C$ ). Therefore, when the drop makes contact with the substrate without additional energy, it remains in the local minimum of free energy level (with the contact angle of $\varphi=180^{\circ}$ ).

When performing experiments in earth conditions, that is, in the acting gravitational field, the deposited drop spreads not only because of the forces acting on interfaces and along the three phase tension line. The gravitational force acting on the droplet is also important because the moving centre 
of gravity of the droplet does the work, which is added to the considered system and helps the liquid to spread over the solid substrate. The larger the drop, the greater the amount of mechanical energy it receives from the surrounding force field. The deformation from the spherical shape resulting from gravity further affects the displacement of the centre of gravity of the liquid, further increasing the mechanical energy added to the system. If this extra portion of energy is greater than that resulting from the energy barrier of point B (Figure 4), the drop will spread on the surface of the substrate "spontaneously" and the experimenter will not know about the "energy given" by the gravitational field. However, if this portion of energy is smaller (corresponding to that between points A and B), then the drop will be "pressed" to the substrate by gravity, thereby deformed from the spherical shape, and although it will not reach a minimum of free energy (but only a state of balance of forces or tensions), the experimenter will be able to measure the contact angle and believe that he has correctly measured it. Only the elimination of the gravitational field could show that the measurement was incorrect, because only in conditions of weightlessness would the drop separate from the substrate and restore its spherical shape. However, if the drop was in thermodynamic equilibrium (point C), during the transition to the state of weightlessness, it would have to "receive" a certain amount of mechanical energy (work) to cross the barrier at point $B$ again, but backward. The rough experimental premise confirming this thesis is included in another paper [35], in which a picture of a small and large drop deposited side by side on a solid substrate is shown. The wetting angle of a small drop is about $150^{\circ}$ and that of a large one is about $110^{\circ}$. The system is influenced by the gravity pressing the drops to the substrate, although more strongly, owing to its mass, a larger drop is pressed. In contrast to a small droplet, the shape of a larger one is significantly different from spherical, and thus it is difficult to attempt a quantitative comparison with the described model.

It should be emphasized that, throughout the entire work, the assumptions and simplifications commonly described in the literature and simultaneously commonly applicable to the thermodynamic description of drops' behaviour in three-phase systems were strictly observed. This also applies to the volume of drops and conditions in which experiments verifying the validity of theoretical predictions were carried out. The presentation of the fact that, for some sets of physicochemical data $\left(\frac{\sigma_{S G}-\sigma_{S L}}{\sigma_{L G}}\right.$ and $\left.\frac{\gamma_{S L G}}{\sigma_{L G} R_{\pi}}\right)$, described models do not meet the sufficient condition of occurrence of the minimum of free energy of the system mainly indicates the imperfections of the previously used hypotheses than the nature of the phenomena under consideration. The presented solutions allow one to assess whether the obtained results of experiments are in the range in which it was possible to obtain the thermodynamic equilibrium of the system. However, reliable values of physicochemical parameters used in the system under test must be known.

The solutions shown in the paper may be a limiting solution for systems in which the surface of a solid substrate is modified (roughness, texture) to improve or to deteriorate its wettability [36,37]. However, such changes in wettability have an impact on the efficiency of microfluidic devices [38,39], which means that a change in the technology for producing microfluidic chips will have an impact on the quality of their operation.

\section{Conclusions}

The results of theoretical considerations based on the principles of non-equilibrium thermodynamics show that, for positive unit energy values of a three-phase tension line (stress line shrinking the wetting circumference), a three-phase system consisting of a spherical drop of liquid and a solid substrate surrounded by a gas may not reach its free energy minimum. This effect is observed for small and large values of contact angles. In addition, for large values of contact angles, the free energy of the system reaches a local maximum, the value of which exceeds the free energy of the drop levitating in the gas. As a result of this phenomenon, a drop deposited on the substrate may spontaneously spread only after exceeding this energy barrier. As the drop spreads over the surface of the solid substrate (decreasing the contact angle), it reaches the minimum of free energy. Further spreading of the drop requires the supply of mechanical energy (work) to it, the value of which is 
higher, the closer the contact angle is to zero; that is, when the liquid covers the solid with a uniform flat layer. It should be emphasized, however, that spontaneous spreading of drops is possible only between the described maximum and minimum values of free energy. In the case when the parameter $G=\frac{\gamma_{S L G}}{\sigma_{L G} R_{\pi}}$ characterizing the physicochemical properties of the system components and volume of liquid, exceeds the value of 3.08 , the drop will not be able to reach the minimum free energy for any value of the contact angle.

For zero and negative unit energy values of the three-phase tension line (stress line stretching the wetting circumference), irrespective of the value of other system parameters, the conditions (necessary and sufficient) of the minimum free energy of a drop deposited on a solid substrate are always met. For negative values of the $G=\frac{\gamma_{S L G}}{\sigma_{L G} R_{\pi}}$ parameter, this takes place even when the solid and liquid phases are strongly repelling themselves at the solid-liquid interface. Moreover, in all such cases, the value of free energy in this minimum is less than the free energy of the spherical drop levitating in gas. This means that the deposition of drop on a solid substrate is a spontaneous process and that, in order to disconnect such a drop from the substrate, it is necessary to provide a certain amount of mechanical energy to it.

Author Contributions: All the authors had equal contribution to the formulation, solution, checking and analysis of the results of the described model.

Funding: This research was funded by Warsaw University of Technology (statutory work) no. 504/03652/7192/40.000101 (J.A.M.) and partially supported by no. 504/03651/7192/40.000101-104 (S.T.) and partially by National Science Centre, Poland grant Opus 8 no. 2014/15/B/ST4/04955 (S.J.).

Conflicts of Interest: The authors declare no conflict of interest.

\section{Appendix A}

Applying the differential form of the equation defining the free energy of the isochoric system, the following relationship can be derived for the system under consideration:

$$
\mathrm{d} F=-S \mathrm{~d} T-\left(p_{L}-p_{G}\right) \mathrm{d} V_{L}+\left(\sigma_{S L}-\sigma_{S G}\right) \mathrm{d} a_{S L}+\sigma_{L G} \mathrm{~d} a_{L G}+\gamma_{S L G} \mathrm{~d} \Lambda_{S L G}
$$

The above relationship was obtained using all substitutions and transformations used to formulate Equations (1)-(7). Only the term describing the entropy of the entire system, $S$, appeared on the right side of Equation (A25) as a result of omitting the isothermal state of the system. However, in the case of an isothermal system, this term is equal to zero owing to $\mathrm{d} T=0$.

The state of thermodynamic equilibrium is defined as the state in which there are no net macroscopic flows of matter or of energy in the whole system or its subsystems (i.e., phases). From the point of view of classical thermodynamics, in the considered system, these conditions will be met when the pressure of vapour of the liquid at the liquid-gas interface is equal to the vapour pressure at any point in the gas phase. This means that the mass of liquid vapour depends on the volume of the gas phase, as well as on the curvature of the liquid-gas interface (Kelvin equation). It is also noteworthy that the conditions of liquid-vapour equilibrium can be met, and the lack of thermodynamic equilibrium of the system results from the inability to achieve a minimum by the sum of interfacial surfaces energy and energy accumulated in the three phase tension line. Exactly this reason is indicated by the analysis of Equation (16). It has to be stressed that, in such a case, failure to meet the thermodynamic equilibrium conditions prevents further analysis of the reasons and impacts of the system behaviour.

To avoid the need to saturate the entire gas phase with vapours, we can benefit from the advantages of non-equilibrium thermodynamics. To do so, an additional, hypothetical thermodynamic cycle has to be introduced near the liquid surface, which will not introduce additional energy to the system under consideration. This condition has to be fulfilled so that no additional non-zero terms appear in Equation (A25). The cycle is performed in a thin, hypothetical layer of gas located just above the surface of the liquid. It consists of four processes: 
(a) evaporation of liquid just on its surface to reach saturated vapour pressure;

(b) isothermal expansion of produced vapours from the liquid surface to vapour pressure in the layer;

(c) isothermal vapour compression from layer pressure to saturated vapour pressure on the liquid surface;

(d) condensation of compressed vapour at saturation pressure on the surface of the liquid.

The work of vapour expansion and work of its compression will compensate when the processes are carried out in a steady state (stationary) and the vapours are treated as perfect gas. In the considered case, the term steady state means that, in each of the processes, the same mass of vapours, independent of time, is involved. Similarly, the heat absorbed and released by the vapours during isothermal expansion and compression will compensate. The heat of liquid evaporation and condensation of vapours will also be compensated. This discussion shows that the hypothetical cycle introduced does not introduce mechanical energy and heat into the system under consideration. Also, its introduction does not create net streams of matter or energy, because all of the processes of the cycle occur in exactly the same place.

During each of the processes included in the cycle, heat is released or absorbed. This means that, according to the second law of thermodynamics, each of these processes will be a source of entropy. Therefore, according to Equation (A25), in order for the increase in entropy not to affect the free energy of the system, it is necessary to assume isothermal $(\mathrm{d} T=0)$ droplet deposition on a solid substrate.

It is noteworthy that the application of non-equilibrium thermodynamics allows one to analyze the free energy behaviour of the system during isothermal drop deposition exactly as it is for non-volatile liquids. In addition, it is possible to track and analyze changes taking place without consideration of liquid evaporation in the droplet volume balance.

\section{References}

1. Young, T. An Essay on the Cohesion of Fluids. Philos. Trans. R. Soc. Lond. 1805, 95, 65-87. [CrossRef]

2. Laplace, P.S. Traite de Mecanique Celeste; Chez Courcier, Imprimeur-Libraire pour les Mathematiques: Paris, France, 1805; Volume 4.

3. Bashforth, F.; Adams, J.C. An Attempt to Test the Theories of Capillary Action; University Press Warehouse: Cambridge, UK, 1883.

4. Maxwell, J.C.; Strutt, J.W. Capillary Action; Encyclopædia Britannica; Cambridge University Press: England, UK, 1911; Volume 5, pp. 256-277.

5. Vesselovsky, V.C.; Pertzov, V.N. Adhesion of Air Bubbles to the Solid Surface. Zhurnal Fiz. Khimii 1936, 8, 245-259.

6. Wenzel, R.N. Resistance of solid surfaces to wetting by water. Ind. Eng. Chem. 1936, 28, 988-994. [CrossRef]

7. Cassie, A.B.; Baxter, S. Wettability of porous surfaces. Trans. Faraday Soc. 1944, 40, 546-551. [CrossRef]

8. Reiss, H. Methods of Thermodynamics; Blaisdell Publishing Company: New York, NY, USA, 1965.

9. Robertson, W.M.; Lehman, G.W. The Shape of a Sessile Drop. J. Appl. Phys. 1968, 39, 1994-1996. [CrossRef]

10. Good, R.J.; Koo, M.N. The Effect of drop size on Contact Angle. J. Colloid Interface Sci. 1979, 71, 283-292. [CrossRef]

11. Schwartz, A.M. The Dynamics of Contact Angle Phenomena. Adv. Colloid Interface 1975, 4, 349-374. [CrossRef]

12. Boruvka, L.; Neuman, A.W. Generalization of the classical theory of capillarity. J. Chem. Phys. 1977, 66, 5464-5476. [CrossRef]

13. Drelich, J.; Miller, J.D. The Line/Pseudo-Line Tension in Three Phase System. Part. Sci. Technol. 1992, 10, 1-20. [CrossRef]

14. Drelich, J. Physicochemical and Engineering Aspects. Colloid Surf. A 1996, 116, 43-54. [CrossRef]

15. Widom, B. Line Tension and the Shape of a Sessile Drop. J. Phys. Chem. 1995, 99, 2803-2806. [CrossRef]

16. Marmur, A.; Krasovitski, B. Line Tension on Curved Surfaces: Liquid Drops on Solid Micro- and Nanospheres. Langmuir 2002, 18, 8919-8923. [CrossRef]

17. Guo, H.K.; Fang, H.P. Drop Size Dependence of the Contact Angle of Nanodroplets. Chin. Phys. Lett. 2005, 22, 787-790. 
18. Letellier, P.; Mayafre, A.; Turmine, M. Drop size effect on contact angle explained by nonextensive thermodymanics. Young's equation revisited. J. Colloid Interface Sci. 2007, 314, 604-614. [CrossRef]

19. Bormashenko, E. Contact angles of rotating sessile droplets. Colloid Surface A 2013, 432, 38-41. [CrossRef]

20. Eral, H.B.; t'Mannetje, J.M. Contact angle hysteresis: A review of fundamentals and applications. Colloid Polym. Sci. 2013, 291, 247-260. [CrossRef]

21. Misyura, S.Y. Contact angle and droplet heat transfer during evaporation on structured and smooth surfaces of heated wall. Appl. Surf. Sci. 2017, 414, 188-196. [CrossRef]

22. Misyura, S.Y. Contact angle and droplet evaporation on the smooth and structured wall surface in a wide range of droplet diameters. Appl. Therm. Eng. 2017, 113, 472-480. [CrossRef]

23. Lubarda, V.A. Mechanics of a liquid drop deposited on a solid substrate. Soft Matter 2012, 40, $10288-10297$. [CrossRef]

24. Lennard-Jones, J.E. On the Determination of Molecular Fields. Proc. R. Soc. Lond. A 1924, 106, 441-477.

25. Saville, G. Computer simulation of the liquid-solid-vapour contact angle. J. Chem. Soc. Faraday Trans. 1977, 73, 1122-1132. [CrossRef]

26. Sikkenk, J.H.; Indekeu, J.O.; Leeuwen, J.M.J.; Vossnack, E.O.; Bakker, A.F. Simulation of wetting and drying at solid-fluid interfaces on the Delft Molecular Dynamics Processor. J. Stat. Phys. 1988, 52, 23-44. [CrossRef]

27. Schmelzer, J. The curvature dependence of surface tension of small droplets. J. Chem. Soc. Faraday Trans. 1986, 82, 1421-1428. [CrossRef]

28. Kanduc, M. Going beyond the standard line tension: Size-dependent contact angles of water nanodroplets. J. Chem. Phys. 2017, 147, 174701. [CrossRef]

29. Marmur, A.; Della Volpe, C.; Siboni, S.; Amirfazli, A.; Drelich, J. Contact angles and wettability: Towards common and accurate terminology. Surf. Innov. 2017, 5, 3-8. [CrossRef]

30. Shapiro, B.; Moon, H.; Garrell, R.L.; Kim, C.J. Equilibrium behavior of sessile drops under surface tension, applied external fields, and material variations. J. Appl. Phys. 2003, 93, 5794-5811. [CrossRef]

31. Tadmor, R. Line Energy and the Relation between Advancing, Receding, and Young Contact Angles. Langmuir 2004, 20, 7659-7664. [CrossRef]

32. Tadmor, R. Line energy, line tension and drop size. Surf. Sci. 2008, 602, L108-L111. [CrossRef]

33. Whyman, G.; Bormashenko, E.; Stein, T. The rigorous derivation of Young, Cassie-Baxter and Wenzel equations and the analysis of the contact angle hysteresis phenomenon. Chem. Phys. Lett. 2008, 450, 355-359. [CrossRef]

34. Erbil, H.Y. The debate on the dependence of apparent contact angles on drop contact area or three-phase contact line: A review. Surf. Sci. Rep. 2014, 69, 325-365. [CrossRef]

35. Saranin, V.A.; Mayer, V.V.; Varaksina, E.I. Investigation of the Shape of a Lying Drop in the Gravity Field and Phenomenon of a Drop Jump during the Transition Weightlessness. Microgravity Sci. Technol. 2018, 30, 121-127. [CrossRef]

36. Drelich, J.; Chibowski, E. Superhydrophilic and Superwetting Surfaces: Definition and Mechanism of Control. Langmuir 2010, 26, 18621-18623. [CrossRef] [PubMed]

37. Li, R.; Shan, Y. Contact Angle and Local Wetting at Contact Line. Langmuir 2012, 28, 15624-15628. [CrossRef]

38. Packham, D.E. Surface energy, surface topography and adhesion. Int. J. Adhes. Adhes. 2003, 23, 437-448. [CrossRef]

39. Gunter, A.; Jhunjhunwala, M.; Thalmann, M.; Schmidt, M.A.; Jensen, K.F. Micromixing of Miscible Liquids in Segmented Gas-Liquid Flow. Langmuir 2005, 21, 1547-1555. [CrossRef]

(C) 2019 by the authors. Licensee MDPI, Basel, Switzerland. This article is an open access article distributed under the terms and conditions of the Creative Commons Attribution (CC BY) license (http://creativecommons.org/licenses/by/4.0/). 